Swansea SWAT/94-95/64

\title{
Random Bond Ising Model and Massless Phase of the Gross-Neveu Model
}

\author{
G. Mussardo' and P. Simonetti \\ Department of Physics and Mathematics, \\ University College of Swansea, \\ Swansea SA2 8PP, UK
}

\begin{abstract}
The $O(n)$ Gross-Neveu model for $n<2$ presents a massless phase that can be characterized by right-left mover scattering processes. The limit $n \rightarrow 0$ describes the on-shell properties of the random bond Ising model.
\end{abstract}

\footnotetext{
${ }^{1}$ Permanent address: International School for Advanced Studies and Istituto Nazionale di Fisica Nucleare, Trieste, Italy.
} 
1. Aim of this paper is to discuss the $S$-matrix formulation of the $O(n)$ Gross-Neveu (GN) model for $n<2$, and in particular the scattering theory associated to the limit $n \rightarrow 0$. There is a well-defined physical problem related to this limit, which is the analysis of the influence of random impurities, induced by thermal fluctuations, on the critical behaviour of pure homogeneous systems. It is therefore useful to briefly remind the relationship between the GN model and the random systems.

For "random temperature" kinds of impurities, i.e. defects or dislocations in the material induced by thermal fluctuations, there is a simple criterion [1] for extimating the relevance of weak disorder on a critical system. According to this criterion, the effect of the disorder depends on the sign of the specific heat critical index $\alpha$ of the pure material. For $\alpha>0$ the impurities are expected to completely suppress the long range fluctuations of the pure system, canceling all singularities in the thermodynamical quantities. On the contrary, for $\alpha<0$, the impurities may produce a shift of the critical temperature but they do not affect the critical behaviour, i.e. the critical exponents are the same as in the pure system. The marginal case $\alpha=0$ is special and must be separately analysed. This situation occurs in the two-dimensional Ising model with random bond distribution, and it has been initially considered by Dotsenko and Dotsenko [2]. In particular, these authors have shown that near the critical temperature, the class of universality of the random bond Ising model is described by the $O(n)$ Gross-Neveu model in the limit $n \rightarrow 0$ (see also [3, 4, 5]). The mapping is realized as follows. The two-dimensional homogeneous Ising model can be described in the continuum limit by a massive Majorana fermion $\Psi$, where the mass $m$ is a linear measurament of the deviation of the temperature $T$ from the critical value $T_{c}$. The partition function for the pure system is given by

$$
Z[m]=\int[d \Psi] \exp \left[-\int d^{2} x \bar{\Psi}(i \not \partial-m) \Psi\right] .
$$

Suppose now that the temperature, instead of being uniform on the whole lattice, is allowed to vary from point to point but staying close, in average, to the critical value. The mass parameter becomes then a random variable $m(x)$, with a probability distribution $P[m(x)]$ which is supposed to be gaussian, $P[m(x)] \sim \exp \left[-m^{2}(x) / 2 g^{2}\right]$. Assuming that the time scale of the spin-flips is much faster than the typical time scale in which the local temperatures are updated, one is led to consider the quenched average on the disorder 
variables, i.e. the average of the free energy rather than the average of the partition function

$$
\overline{\ln Z[m(x)]}=\int \ln Z[m(x)] P[m(x)] d m(x) .
$$

A standard method to get around the difficulty presented by this computation is to use the replica trick [6], which consists in replacing

$$
\ln Z \rightarrow \lim _{n \rightarrow 0} \frac{Z^{n}-1}{n}
$$

in the integral of the right hand side of (2). In this way, taking the quenched average for the random bond Ising model is equivalent to solve the dynamics of the Gross-Neveu model in the limit $n \rightarrow 0$

$$
\overline{\ln Z} \sim \int\left[d \Psi_{i}\right] \exp [-\mathcal{S}]
$$

where

$$
\mathcal{S}=\int d^{2} x\left[\bar{\Psi}_{i} \not \partial \Psi_{i}+\frac{g^{2}}{2}\left(\bar{\Psi}_{i} \Psi_{i}\right)^{2}\right] \quad, \quad i=1, \ldots, n
$$

2. It is well known that the Gross-Neveu model [7] presents different properties for $n>2$ and for $n<2$ ( for $n=2$ it coincides with the massless Thirring model, which is a Conformal Field Theory). This can be seen, for instance, by considering the $\beta$ function of the theory, that at the first order reads [8]

$$
M \frac{d}{d M} g(M)=\beta(g(M))=-(n-2) \frac{g^{3}}{2 \pi}+\cdots
$$

( $M$ is a mass scale). For $n>2$ the theory is asymptotically free in the ultraviolet limit and there is a dynamical mass generation. The chirality symmetry of the original lagrangian (4) is spontaneously broken and the fermionic fields are actually massive. The resulting theory is integrable and the exact elastic $S$-matrix has been computed in [9, 10]. In addition to the elementary fermions appearing in the lagrangian (4), the final spectrum of the theory comes out to have quite a rich structure of bound states.

The situation is rather different when $n<2$. Since the $\beta$ function is now positive around the origin, the GN model becomes an infrared asymptotically free theory and therefore a dynamical mass generation for the physical particles cannot occur [7]. In this case, we can extract the spectrum by directly looking at the infrared limit of the lagrangian 
(4): it simply consists in $n$ copies of massless Majorana fermions and no additional lowenergy excitations are expected. Since the $\beta$-function is not zero, the model is massless but not conformally invariant and therefore the mass scale $M$ entering the Renormalization Group equation (5) characterises the cross-over of the theory going from the infrared to the ultraviolet region. This massless phase of the GN model consists then of $n$ right-moving particles $R_{a}(p)$ with the energy spectrum $E=p(p>0)$ and $n$ left-moving particles $L_{a}(p)$ $(p<0)$ with $E=-p$. These dispersion relations can be conveniently parameterized in terms of the mass scale $M$ and the rapidity variable $\theta$ as follows: $E=p=\frac{M}{2} e^{\theta}$ for the right-movers and $E=-p=\frac{M}{2} e^{-\theta}$ for the left-movers.

3. Assuming that the integrability of the GN model also holds for $n<2$, we can compute the exact elastic $S$-matrix associated to this massless phase of the model (for the definition of massless scattering and its properties, we refer the reader to the original references [11, 12]). In the scattering processes of integrable massless theories, the numbers of left and right particles are separately conserved and there is a factorization of the amplitudes. Hence we can restrict our attention only to the two-body processes. For the infrared asymptotic freedom of the model, we expect no scattering of the left-left or right-right particles. Therefore, in these two sectors the $S$-matrix is simply -1 . On the contrary, in the right-left sector, the $S$-matrix is defined by the commutation relation

$$
R_{a}\left(\theta_{1}\right) L_{b}\left(\theta_{2}\right)=S_{a b}^{c d}\left(\theta_{1}-\theta_{2}\right) L_{d}\left(\theta_{2}\right) R_{c}\left(\theta_{1}\right)
$$

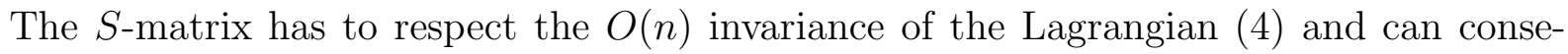
quently be decomposed as

$$
S_{a, b}^{c, d}(\theta)=\delta_{a b} \delta^{c d} \sigma_{1}(\theta)+\delta_{a}^{c} \delta_{b}^{d} \sigma_{2}(\theta)+\delta_{a}^{d} \delta_{b}^{c} \sigma_{3}(\theta)
$$

Equivalently, we may consider the $S$-matrix for the channels of definite isospin,

$$
\begin{aligned}
& \sigma_{\text {isoscalar }}=N \sigma_{1}+\sigma_{2}+\sigma_{3} ; \\
& \sigma_{\text {antisym }}=\sigma_{2}-\sigma_{3} ; \\
& \sigma_{\text {sym }}=\sigma_{2}+\sigma_{3} .
\end{aligned}
$$


The above amplitudes have to satisfy the unitarity and crossing symmetry equations, which for the massless scattering are given respectively by

$$
\begin{gathered}
\sigma_{2}(\theta) \sigma_{2}^{*}(\theta)+\sigma_{3}(\theta) \sigma_{3}^{*}(\theta)=1 ; \\
n \sigma_{1}(\theta) \sigma_{1}^{*}(\theta)+\sigma_{1}(\theta) \sigma_{2}^{*}(\theta)+\sigma_{1}(\theta) \sigma_{3}^{*}(\theta)+\sigma_{2}(\theta) \sigma_{1}^{*}(\theta)+\sigma_{3}(\theta) \sigma_{1}^{*}(\theta)=0 ; \\
\sigma_{2}(\theta) \sigma_{3}^{*}(\theta)+\sigma_{3}(\theta) \sigma_{2}^{*}(\theta)=0
\end{gathered}
$$

and

$$
\begin{aligned}
& \sigma_{1}^{*}(\theta)=\sigma_{3}(i \pi+\theta) \\
& \sigma_{2}^{*}(\theta)=\sigma_{2}(i \pi+\theta) .
\end{aligned}
$$

The associativity condition for the algebra (6) gives rise to the Yang-Baxter equations, whose solution is given by

$$
\begin{aligned}
\sigma_{1}(\theta) & =-\frac{i \lambda}{i \lambda\left(\frac{n-2}{2}\right)-\theta} \sigma_{2}(\theta) ; \\
\sigma_{3}(\theta) & =-\frac{i \lambda}{\theta} \sigma_{2}(\theta)
\end{aligned}
$$

with the parameter $\lambda$ fixed by the crossing symmetry equations (10) to be

$$
\lambda=\frac{2 \pi}{n-2}
$$

Inserting (11) into eq. (9) and using (10), we arrive to the combined unitarity-crossing equation satisfied by $\sigma_{2}$,

$$
\sigma_{2}(\theta) \sigma_{2}(i \pi+\theta)=\frac{\theta^{2}}{\theta^{2}+\lambda^{2}}
$$

The minimal solution is given by

$$
\sigma_{2}(\theta)=-\frac{\Gamma\left(1-\frac{\theta}{2 \pi i}\right) \Gamma\left(\frac{1}{2}+\frac{\theta}{2 \pi i}\right) \Gamma\left(\frac{1}{2}-\frac{\lambda}{2 \pi}-\frac{\theta}{2 \pi i}\right) \Gamma\left(-\frac{\lambda}{2 \pi}+\frac{\theta}{2 \pi i}\right)}{\Gamma\left(\frac{\theta}{2 \pi i}\right) \Gamma\left(\frac{1}{2}-\frac{\lambda}{2 \pi}+\frac{\theta}{2 \pi i}\right) \Gamma\left(\frac{1}{2}-\frac{\theta}{2 \pi i}\right) \Gamma\left(1-\frac{\lambda}{2 \pi}-\frac{\theta}{2 \pi i}\right)} .
$$

This expression coincides with the analogous formula obtained in the GN model for $n>2$ but there is an important difference here: for $n<2, \lambda$ is negative and therefore the amplitudes present no poles in the physical strip. The absence of poles in the physical strip matches of course with the nature of massless scattering. It is easy to see that for $n=1$ (which is simply the massless Ising model) the isoscalar $S$-matrix in (8) correctly reduces to -1 whereas for $n \rightarrow 0$, the relevant limit for the random bond Ising model, we 
have

$$
\sigma_{2}(\theta)=-\frac{\theta}{(i \pi-\theta)}\left(\frac{\Gamma\left(\frac{1}{2}+\frac{\theta}{2 \pi i}\right) \Gamma\left(-\frac{\theta}{2 \pi i}\right)}{\Gamma\left(\frac{1}{2}-\frac{\theta}{2 \pi i}\right) \Gamma\left(\frac{\theta}{2 \pi i}\right)}\right)^{2} .
$$

In conclusion, our analysis shows that for $n<2$ the $S$-matrix of the elementary fermions of the GN model may be regarded as the analytic continuation of the $S$-matrix for $n>2$ and viceversa. The interpretation, however, is quite different in the two intervals. In fact, for $n>2$ the $S$-matrix describes the interaction of massive fermions and, in order to close the bootstrap, has to be supported by the computation of the other scattering amplitudes relative to the bound states [9, 10]. For $n<2$, on the contrary, there are no bound states, the fermionic fields split into the chiral components and the $S$-matrix describes in this case only the interaction of the left-right moverst.

4. The massless $S$-matrix proposed in this paper leaves open some important questions. The first question concerns the characterization of the ultraviolet limit of the GN model for $n<2$. In fact, the perturbative approach to an infrared free theory is usually plagued by the presence of Landau pole which may prevent to extend the validity of the theory beyond it. If this is the case, the ultraviolet region cannot be reached perturbatively from the infrared fixed point. The massless GN $S$-matrix (14) has no singularities for real values of $\theta$. This seems to indicate that for the massless GN model, the ultraviolet limit is, instead, continuosly connected to the infrared fixed point. In order to clarify this point, a first step would be to analyse the behaviour of the massless GN model on an infinite cylinder of width $R$ and extract, by means of the Thermodynamical Bethe Ansatz, the flow of the effective central charge $\tilde{c}(M R)$ between the ultraviolet $(M R \rightarrow 0)$ and the infrared $(M R \rightarrow \infty)$ fixed points. The second question concerns the local properties of the GN model and their relationship with the original statistical system described by the limit $n \rightarrow 0$. As for the limit $n \rightarrow 0$ of the bosonic $O(n)$ model studied in [13, 14] in connection to the self-avoiding walks, it would be interesting to apply the massless Form Factor approach, recently considered in [15], and study the behaviour of the correlation functions of the random bond Ising model associated to the $S$-matrix of eq. (15).

\footnotetext{
${ }^{1}$ Obviously, also the interpretation of the rapidity variable changes in the two intervals.
} 
Acknowledgements. We are grateful to G. Delfino, J.L. Cardy, T. Hollowood and A. Schwimmer for useful discussions. One of us (GM) thanks Prof. D.I. Olive and the Theoretical Physics Department of University College of Swansea for warm hospitality.

\section{References}

[1] A.B. Harris, J. Phys. C 7 (1974), 1671.

[2] Vi.S. Dotsenko and Vl.S. Dotsenko, JETP Lett. 33 (1981), 37; Adv. in Phys. 32, (1983), 129.

[3] B.N. Shalaev, Sov. Phys. Solid State 26 (1984), 1811.

[4] R. Shankar, Phys. Rev. Lett. 58 (1987), 2466; Phys. Rev. Lett. 61 (1988), 2390.

[5] A.W.W. Ludwig, Phys. Rev. Lett. 61 (1988), 2388; H.A. Ceccatto and C. Naon, Phys. Rev. Lett. 61 (1988), 2389.

[6] S.F. Edwards and P.W. Anderson, J. Phys. F 5 (1975), 965.

[7] D.J. Gross and A. Neveu, Phys. Rev. D 10 (1974), 3235.

[8] W. Wetzel, Phys. Lett. B 153 (1985), 297.

[9] A.B. Zamolodchikov and Al.B. Zamolodchikov, Ann. Phys. 120 (1979), 253.

[10] M. Karowski and H.J. Thun, Nucl. Phys. B 130 (1977), 224,

[11] Al. B. Zamolodchikov, Nucl. Phys. B 358, 524 (1991).

[12] A.B. Zamolodchikov and Al.B. Zamolodchikov, Nucl. Phys. B 379 602, (1992); P. Fendley, H. Saleur and Al. B. Zamolodchikov, Int. J. Mod. Phys. A 8, 5751 (1993).

[13] A.B. Zamolodchikov, Mod. Phys. Lett. A 6 (1991), 1807.

[14] J.L. Cardy and G. Mussardo, Nucl. Phys. B 410 (1993), 451.

[15] G. Delfino, G. Mussardo and P. Simonetti, Correlation Functions along a Massless Flow, hep-th 9410117. 